\title{
A Improved Video Codec Supporting Error Resilience and Resource Adaptation
}

\author{
Praveen Sanigepalli ${ }^{\dagger}$, Hari Kalva ${ }^{\ddagger}$, Borko Furht ${ }^{\ddagger}$ \\ ${ }^{\dagger}$ Motorola, Plantation, FL $\quad$ 'Florida Atlantic University, Boca Raton, FL
}

\begin{abstract}
Mobile phones have become essential part of modern life and continue to change the way people communicate with each other. The camera phones have become ubiquitous in the recent past and video communication services such as videophone are getting rolled out by cellular providers. This would require video compression algorithms that are resilient to transmission errors and support resource-adaptive playback. In this paper, we propose a video codec that addresses the two key issues in video delivery to mobile devices: 1) error resilience and 2) resource adaptive playback. We show that the proposed codec is simple to implement on low-resource terminals such as mobile phones and outperforms MPEG-4 coding in terms of loss tolerance.
\end{abstract}

\section{INTRODUCTION}

Current research on Video Compression algorithms focus on optimizing the efficiency [4]. Research in the area of error resilience of compressed video streams target errors over the air or network and do not take into account the resource constraints of devices [1,5]. A resource constrained mobile device may have to drop video frames prior to decoding, thus resulting in error propagation due to the use of incorrect reference frames. It is essential to devise video compression algorithms that not only support error resilience but also enable video adaptation at the receiver for constraints such as CPU capacity and available power. In this paper, we propose a video codec that addresses the two key issues in video delivery to mobile devices: 1) error resilience and 2) resource adaptive playback. The proposed video codec reduces the prediction dependencies present in traditional MPEG coding to enable error resilience. We evaluate the performance of the codec for different frame loss rates. We show that the proposed method performs significantly better compared to traditional MPEG coding.

All the MPEG video coding standards use hybrid video coding that exploits spatial and temporal redundancies to achieve high compression rates. The predictive coding techniques used in MPEG video create frame dependencies that result in propagation of any errors that are a direct result of losses in the network or frame dropping at the receiver. H.263+ specifies a reference picture selection (RPS) mode where a past frame is referenced at the encoder for prediction if decoder reference frame is distorted [2]. However, such a technique requires feedback from the decoder channel that is not available in broadcast or multicast applications. While
H.264 supports improved error resilience through multi-frame prediction [3], it is considered complex to be implemented on mobile devices today and MPEG-4 or H.263 is preferred.

\section{PROPOSED SCHEME}

In this paper we propose a new scheme, periodic intraframe based prediction (PIFBP), in which all the P frames in a group of pictures (GOP) use the same I-frame as a reference - see Figure 1. This technique localizes errors in $\mathbf{P}$ frames to just those frames and eliminates distortion propagation. This improves error resilience but with a loss in quality. Our experimental results indicate that the resulting drop in quality is not substantial and provides significant error resilience performance. This method can also be realized as a special case of H.264 video coding.

Figure 2 shows the performance of PIFBP scheme over MPEG-4 Simple Profile (SP) codec under frame loss conditions. The frame drop simulates two conditions: 1) frame drop because of bit errors and packet loss in the wireless network and 2) frame drop at the receiver due to limited battery or computing resources. Foreman sequence was used for the simulation and the frames are dropped randomly. The periodicity of I frames is varied and the frame loss was simulated. For PIFBP, the period of I frames is chosen as 5. Figure 3 shows the plot of PSNR values of each individual frames of foreman sequence encoded using PIFBP and MPEG-4 SP profile codecs. The sequence is encoded at the rate of $128 \mathrm{kbps}$ and frame loss of $15 \%$ is simulated. With MPEG-4 baseline codec, the drop in quality corresponds to a loss of a previous frame whereas PIFBP scheme maintains consistent quality despite frame loss. These results demonstrate that this scheme maintains the same quality under frame loss and performs really well.

PAFBP (Periodic anchor based frame prediction) is a variation of PIFBP scheme. In this scheme, an I frame is encoded as an anchor frame at start and periodically anchor frames are encoded using prediction from previous anchor frames. Within the GOP period, the $\mathrm{P}$ frames are predicted from the anchor frame. For the foreman sequence, the PAFBP scheme improved PSNR by approximately $1 \mathrm{db}$.

\section{CONCLUSION}

Extensive simulation of the scheme has been performed and the scheme outperforms MPEG-4 Simple Profile. The 
different error resilience techniques that are part of the MPEG-4 standard do not improve the performance and do not address the issues of resource-constrained devices. The scheme is applicable in several use cases such as random access into MPEG-4 stream, content adaptation by intermediate points, and congestion management in jitter buffer for packet data networks. This scheme presents an efficient and effective solution for mobile video delivery.

\section{REFERENCES}

[1] Yao Wang and Qin-Fan Zhu, "Error Control and Concealment for Video Communications: A Review", Proceedings of the IEEE, Vol. 86, NO. 5, May. 1998.
[2] Chun g-How, J.T.H. and Bull, D.R., "Robust H.263+ video for Real-Time Internet Applications", Proceedings of International Conference on Image Processing, vol. 3, pp. 544-547, Sept. 2000.

[3] Stockhammer, T., Hannuksela, M.M., and Wiegand, T.," H.264/AVC in wireless environments", IEEE Transactions on Circuits and Systems for Video Technology, Volume: 13, Issue: 7, July 2003 Pages: $657-673$

[4] G. J. Sullivan and T. Wiegand, "Rate-distortion optimization for video compression," IEEE Signal Proc. Magazine, Vol. 15, no. 6, pp. 74-90, Nov. 1998

[5] Brailean James, "Wireless Multimedia utilizing MPEG-4 Enor resilient Tools", IEEE Transactions, 1999.

[6] Jun Wang, Sinnarajah, R., Tao Chen, Yongbin Wei, Tiedemann, E. "Broadcast and multicast services in coma2000", IEEE Communications Magazine, Vol: 42, Issue: 2, Page(s):76- 82, Feb. 2004.

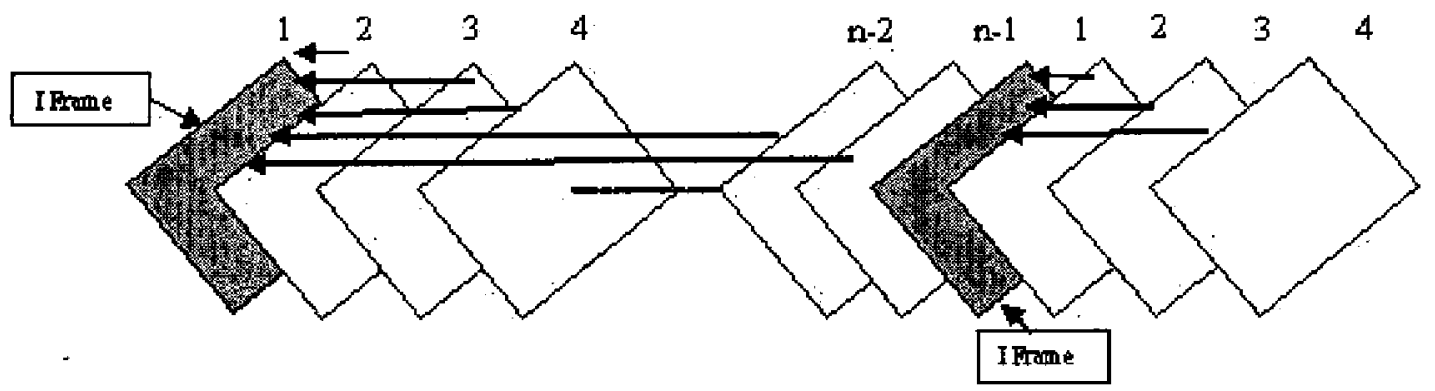

Figure 1 Periodic Intra frame based prediction (PIFBP)

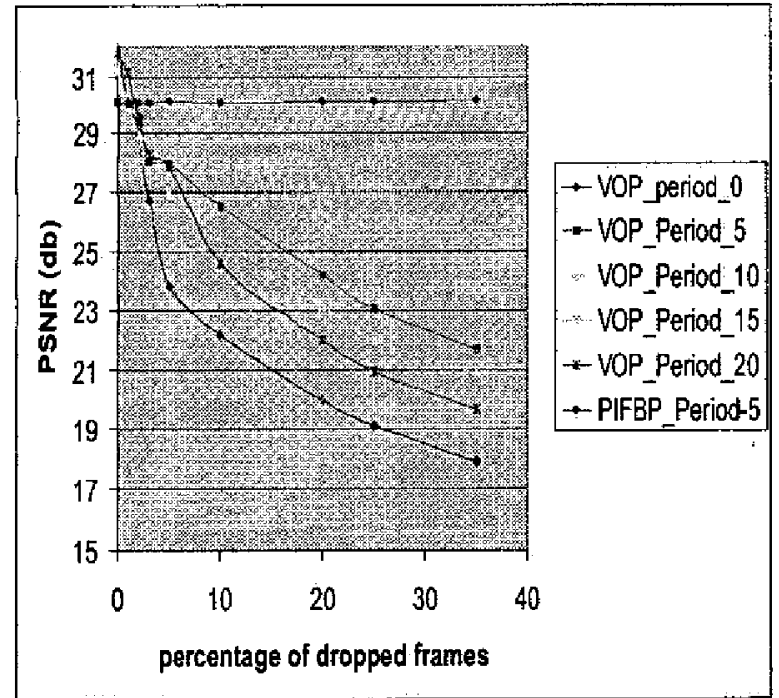

Figure 2 Performance under loss of Inter / Intra frame coding (Data rate $=128 \mathrm{kbps}, \mathrm{fps}=15 \mathrm{fps}$ )

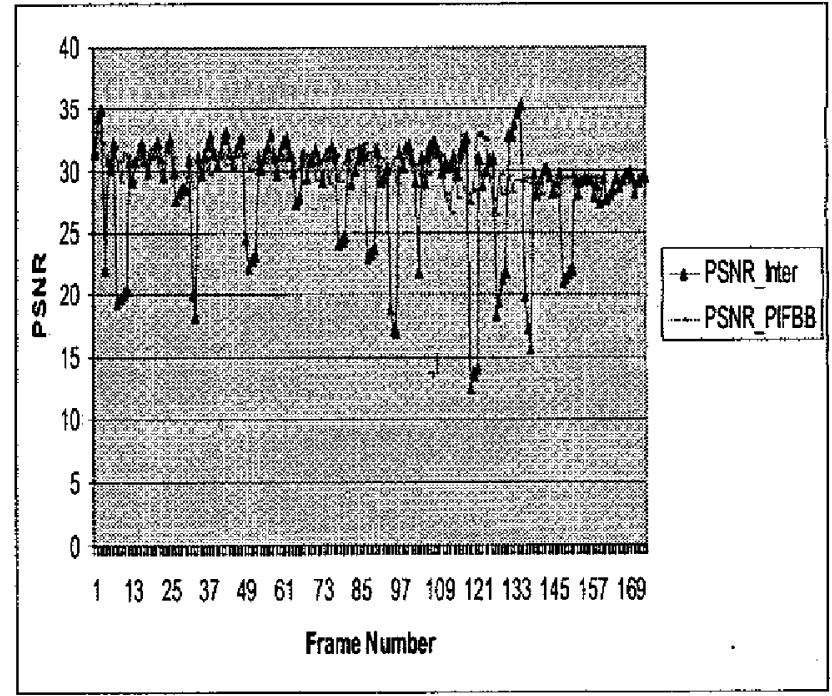

Figure 3 Comparison of individual frame PSNR's for PIFBP and MPEG-4 schemes with VOP periodicity of 5 under $15 \%$ frame loss 